\title{
Simulation Analysis of Atomic Scale Optical Imaging by Combing Photoexcitation and Electron Microscopy
}

\author{
Ze Zhang ${ }^{1}$, Archith Rayabharam ${ }^{2}$, Joel Martis ${ }^{1}$, Haokun Li $^{1}$, Narayana Aluru ${ }^{2}$ and Arun Majumdar ${ }^{1}$ \\ ${ }^{1}$ Stanford University, Stanford, California, United States, ${ }^{2}$ UIUC, Urbana, Illinois, United States
}

Optical imaging with atomic scale resolution offers fundamental insights in field such as quantum information, catalysis, optoelectronics and structural biology [1-3]. It provides rich information about the electronic or vibrational states of the material system, which greatly enhances our ability to understand and design novel material systems. Optical spectroscopy and imaging techniques like Raman, photoluminescence and IR spectroscopy are already widely used for materials characterization. However, their spatial resolution is limited by the wavelength of light used, which is hundreds of nanometers to microns. Although various super-resolution techniques have been developed and achieved about $10 \mathrm{~nm}$ resolution, sub-nanometer and atomic scale optical imaging still remains elusive [4-5].

Here we propose a new imaging technique named PhotoAbsorption Microscopy using ELectron Analysis (PAMELA), which combines the high spectrally specific photoabsorption and the high spatial resolution of electron beams to obtain optical imaging at nanometer and atomic scale. Figure 1 presents our PAMELA set-up, where we developed a transmission electron microscope (TEM) holder with optical fiber to optically excite the sample in-situ during high resolution TEM imaging. Modern aberration corrected high resolution (HR-) TEM can achieve sub angstrom resolution readily, and the contrast are mainly phase contrast in HRTEM. Upon optical excitation, the electron density is redistributed in the optical active area, while other areas are unaffected. This change of charge distribution and electrical potential will produce atomic scale contrast in the final HRTEM image. To further enhance the signal-tonoise ratio, we developed imaging lock-in algorithms to help detect the small signal change in the HRTEM image with photo excitation.

We choose atomic scale defects in monolayer hexagonal Boron Nitride (h-BN) as the first material system to demonstrate PAMELA technique. Monolayer two-dimensional (2D) materials are clean model systems for TEM imaging and minimize the complication from multiple scattering. Also defects in $\mathrm{h}-\mathrm{BN}$ are optical active and are room-temperature single photon emitters, which are of great interest for quantum information application [6-7]. Given the wide interest, the correlation between the atomic structure and optical properties are not well understood due to lack of tools for optical imaging at atomic scale.

To simulate the HRTEM imaging change with photoexcitation in h-BN defects, we first calculate the electron wave function and charge density distribution around local defects in the ground state and excited state using density functional theory (DFT). From the charge distribution, we calculate the electrical potential of the sample, and perform the HRTEM simulation using the multi-slice method. Different defocus and aberration coefficient values are used to compute the final HRTEM image (Figure 2a-b). It is clear that there is a compromise between contrast and spatial resolution. We choose $10 \mathrm{~nm}$ defocus and $15 \mu \mathrm{m}$ spherical aberration coefficient value, and add shot noise and camera noise to the calculation. As shown in Figure 2c, with 16 min data acquisition, the difference between the ground state and excited state starts to be visible in the final HRTEM image.

In summary, we proposed a new imaging technique PAMELA which combines optical excitation and electron microscopy to achieve atomic scale optical imaging. The feasibility has been discussed using 
DFT and HRTEM simulations. As it relies on the inherent light absorption and electron charge redistribution, it could be a more general approach for material characterization. This will open new opportunities in various application such as defects engineering, heterogenous catalysis, biosensing and imaging.

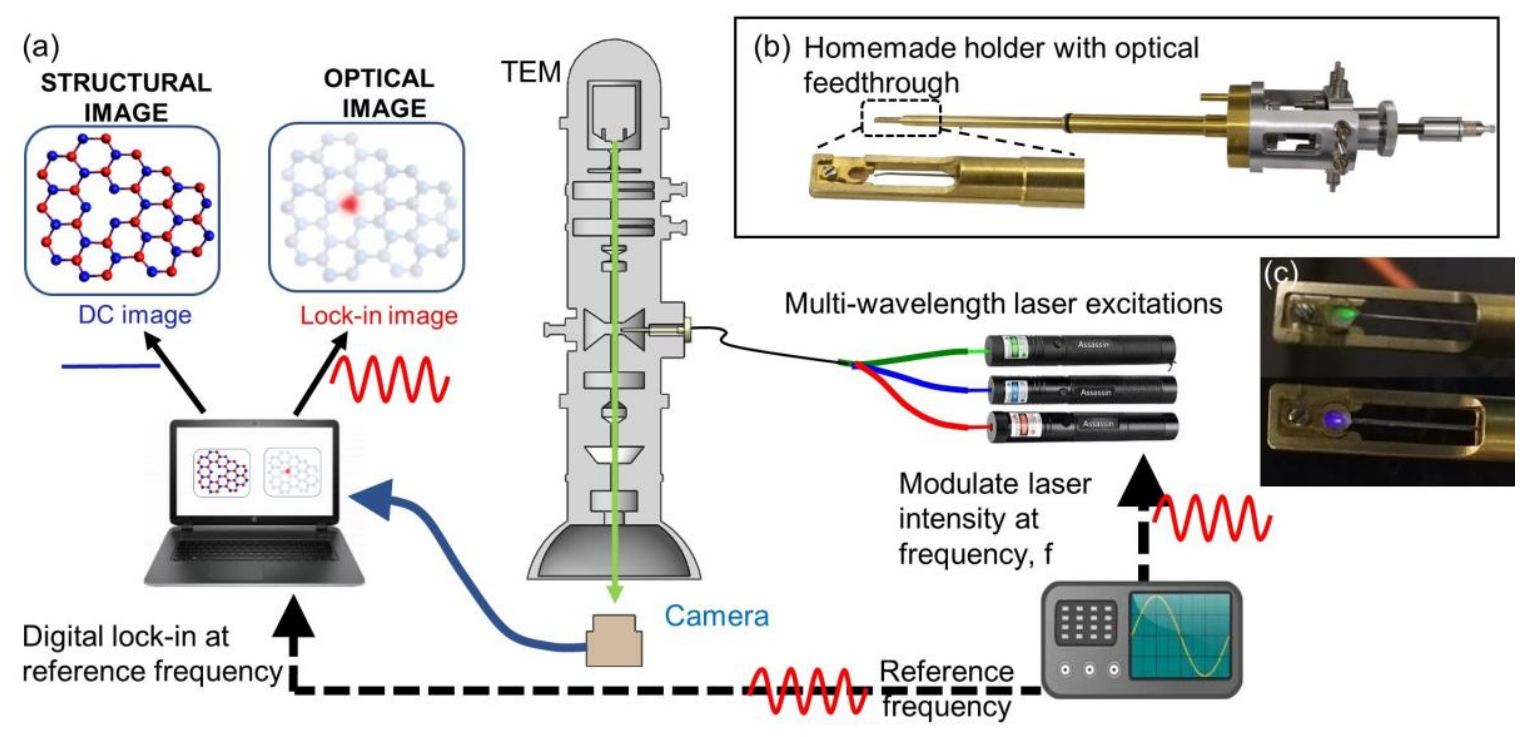

Figure 1. (a) Schematic illustration of the PAMELA technique we are developing, which aims for optical image at atomic scale. The sample is optically excited with multi-wavelength lasers. The intensity of these lasers are modulated and the images are lock-in at the same frequency to enhance signal-to-noise ratio. Structural information is obtained by the averaged DC image, while optical information is obtained by the lock-in image. (b) A homemade TEM holder with optical feedthrough to optical excite the sample during HRTEM imaging. (c) Images of the holder tip with two different wavelengths of laser excitations. 
(a)

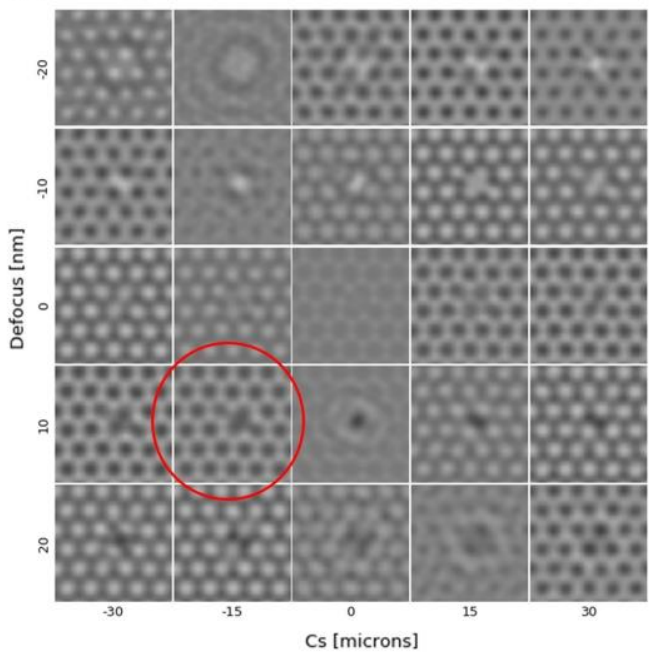

(c) Dose: $1 \mathrm{e} 6 \mathrm{e}-/ \AA^{2}$
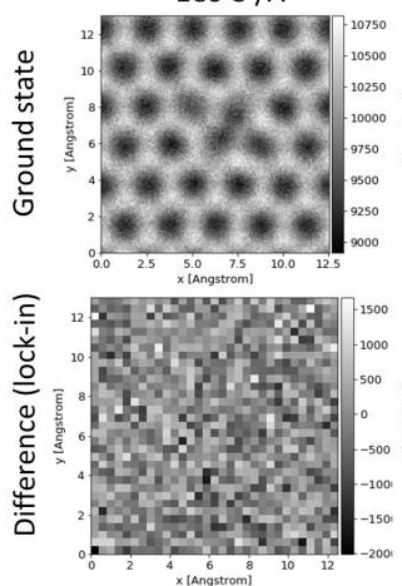

Ave. time: 100 s

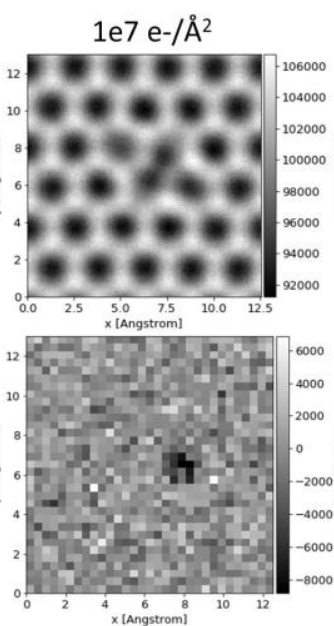

$16 \mathrm{~min}$ (b) Difference (lock-in) images

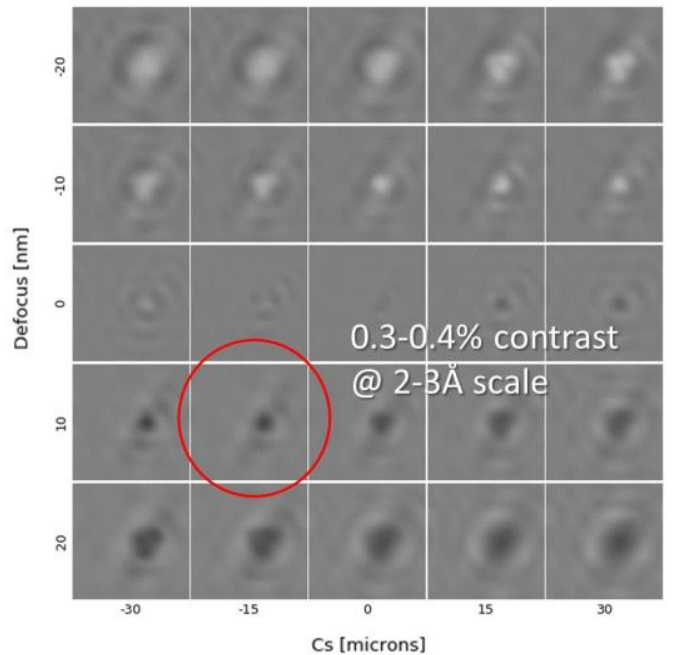

$1 \mathrm{e} 8 \mathrm{e}-/ \AA^{2}$
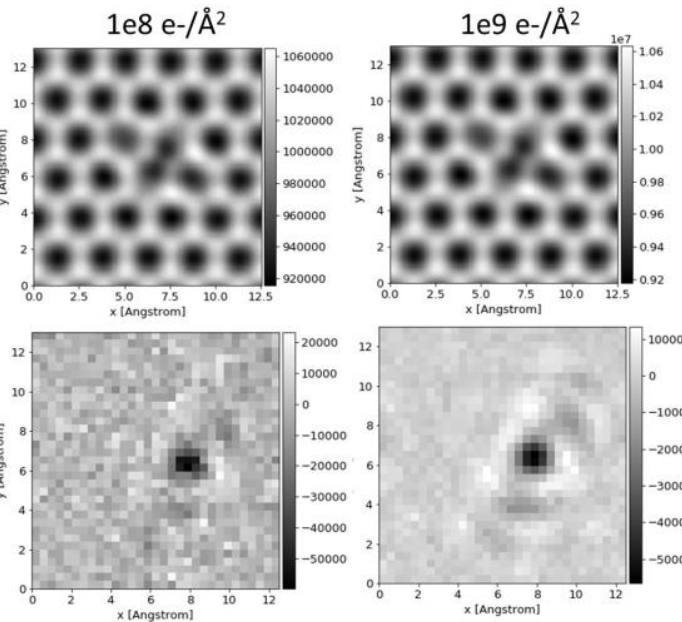

$2 \mathrm{~h} 40 \mathrm{~min}$

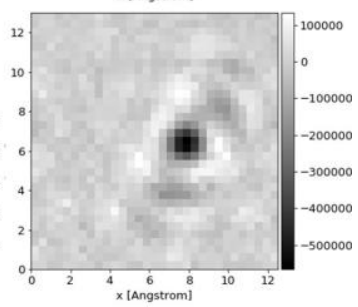

$>1$ day

Figure 2. TEM simulations of a missing BN defect in monolayer h-BN. (a) structural image under different defocus and aberration coefficient. (b) optical image obtained by calculating the difference between the excited and ground state images. Red circled image shows the condition we use for simulation with shot noise in (c). The top row images are for ground state and the bottom row images are for the difference between excited and ground state. Different column shows different dose (1e6 -- 1e9 e-/A2) and corresponding acquisition time (100 s - 1 day) required.

\section{References}

[1] Aharonovich, Igor, Dirk Englund, and Milos Toth. "Solid-state single-photon emitters." Nature Photonics 10.10 (2016): 631.

[2] Hong, Jinhua, et al. "Atomic Defects in Two-Dimensional Materials: From Single-Atom Spectroscopy to Functionalities in Opto-/Electronics, Nanomagnetism, and Catalysis." Advanced Materials 29.14 (2017): 1606434.

[3] Hou, Wenbo, and Stephen B. Cronin. "A review of surface plasmon resonance-enhanced photocatalysis." Advanced Functional Materials 23.13 (2013): 1612-1619. 
[4] Harootunian, A., et al. "Super-resolution fluorescence near-field scanning optical microscopy." Applied Physics Letters 49.11 (1986): 674-676.

[5] Galbraith, Catherine G., and James A. Galbraith. "Super-resolution microscopy at a glance." Journal of cell science 124.10 (2011): 1607-1611.

[6] Huang, Bing, and Hoonkyung Lee. "Defect and impurity properties of hexagonal boron nitride: A firstprinciples calculation." Physical Review B 86.24 (2012): 245406.

[7] Tran, Toan Trong, et al. "Quantum emission from hexagonal boron nitride monolayers." Nature nanotechnology 11.1 (2016): 37. 